\title{
El impacto socioeconómico de las políticas de buen gobierno de las entidades de economía social en España
}

\author{
Socio-economic impact of corporate governance \\ policy statement for social economy in Spain
}

María de Carmen Muñoz Medraño'
Francisco Laborda Peñalver ${ }^{2}$
Antonio Juan Briones Peñalver ${ }^{3}$

Fecha de recepción: 20 de enero del 2012

Fecha de aprobación: 19 de mayo del 2012

Muñoz, M; Laborda, F; Briones, A. El impacto socioeconómico de las políticas de buen gobierno de las entidades de economía social en España. Tecnología en Marcha. Vol. 25, № 6. Pág 29-35.

I Máster por la Universidad Politécnica de Cartagena en Administración y Dirección en Entidades de la Economía Social. Investigadora del Grupo de Investigación Estrategias Organizativas y Comerciales de las Empresas. Correo electrónico: maicamu@hotmail.com

2 Catedrático de Escuela Universitaria, Universidad Politécnica de Cartagena. Correo electrónico: francisco.laborda@upct.es

3 Profesor Colaborador de la Facultad de Ciencias de la Empresa, Coordinador Proyecto Financiado por el Programa de Cooperación Interuniversitaria e Investigación Científica PCI-AECID A/032048/I0 Correo electrónico: aj.briones@upct.es 


\section{Palabras clave}

Gobierno, desarrollo económico, empleo, integración, economía social.

\section{Resumen}

Las entidades de la economía social que representan el sector empresarial de mercado, con prácticas de buen gobierno en sus sistemas directivos, favorecen positivamente el impacto socioeconómico en los territorios donde se asientan. En este trabajo se identifican que en las prácticas directivas de estas entidades, confluyen principios democráticos en pro del buen gobierno y de las economías solidarias, posibilitando la creación de más empleo estable y de calidad, en comparación con otro tipo de organizaciones empresariales tradicionalmente analizadas como de economía capitalista. Tras la revisión de la literatura se pone en relieve actualizadas cifras del país en lo referente al impacto socioeconómico y en particular los datos del empleo generado, el desarrollo local en zonas rurales, así como la integración socio-laboral.

\section{Key words}

Government, stakeholders, strategies, results, social economy.

\begin{abstract}
The corporate governance of Social economy entities (hereinafter, EES) improves the efficiency and growth of these organizations with or without profit through strategic measures. The incidence of this model of governance is basic in the EES and through stakeholders or interest group provides integration for the different actors involved in socio-economic relations. In this sense, theories and traditional approaches to business management point out the EES such as those that contribute to its institutions and actors, innovative nature for the best territorial and socio-economic development of the peoples where settle.
\end{abstract}

\section{Introducción}

La situación de enormes dificultades que se está atravesando en general en todas las economías ha provocado la atención de los agentes socioeconómicos en aquellos aspectos relativos a la mejora de las necesidades sociales, corrección de los desequilibrios económicos y un pretendido aumento de la búsqueda de confianza por parte de las empresas en los mercados financieros. Ello hace que las empresas lleven a cabo cada vez más prácticas encaminadas al buen gobierno, en parte debido a la gran presión social y a que los ciudadanos valoren positivamente la implantación de políticas en este sentido.

La economía social (en adelante ES) es un buen ejemplo de esta nueva forma de hacer empresa, en asuntos relacionados con su gobierno y el impulso socioeconómico que conlleva. La gestión democrática de estas entidades, basada en sus principios y sus valores propios, les lleva a desarrollar su actividad de manera sostenible y responsable, repercutiendo positivamente, no solo en la organización, sino también en la sociedad y en los agentes (stakeholders) con los cuales se relaciona.

De ahí que en los últimos años las entidades de la ES (en adelante EES), identificadas por el Ministerio de Trabajo e Inmigración como "sociedades laborales y cooperativas" se han consolidado como un fuerte pilar de desarrollo socioeconómico para la sociedad española; por ello, su estudio suscita gran interés entre los investigadores. Organizaciones como CEPES (Confederación Empresarial Española de la Economía Social) o CIRIEC-España (Centro Internacional de Investigación e Información sobre la Economía Pública, Social y Cooperativa) han elaborado múltiples estudios destinados a medir el peso de la ES, sus grandes cifras, su aportación al empleo y su importancia en los distintos sectores de actividad donde las EES están presentes.

En este artículo se hará un análisis de algunos datos que arrojan los distintos estudios y estadísticas que 
permitirán estudiar la importancia de las EES en España así como el impacto socioeconómico de sus políticas de buen gobierno en la sociedad española. Además, los datos empleados en esta investigación forman parte de un análisis previo que se está llevando a cabo para sentar los antecedentes de un estudio más amplio sobre el buen gobierno y sus prácticas.

El artículo se divide en varias partes. En la primera se lleva a cabo un análisis de la literatura sobre lo que los investigadores entienden por políticas de buen gobierno en la dirección estratégica, las prácticas basadas en sus principios y los valores cooperativos. Se analiza con datos el incremento de las EES en su conjunto y la representatividad que tiene en España, así como la importancia que tiene en el conjunto de la economía europea.

Después se analiza prioritariamente el impacto socioeconómico de las políticas de gobierno en estas EES, la integración social o laboral proporcionada entre los agentes (stakeholders) con los que se relacionan, la creación de empleo estable, de calidad y el desarrollo local proporcionado en las zonas rurales donde particularmente se asientan algunas de las organizaciones empresariales.

\section{El gobierno en las EES}

El gobierno corporativo en las EES es considerado una forma de dirección eficiente en el uso de los activos que las configuran (Bueno, 2004); se asienta en principios de solidaridad y prácticas de democracia económica (Juliá, 2004; Chaves, 2004); ofrece información fiable a los stakeholders (Seguí et al., 2008) y en general, dota de fortalezas a las organizaciones que siguen estas premisas (Saz y Carús, 2008).

Según la Organización para la Cooperación y el Desarrollo Económico el buen gobierno, es un "elemento clave para mejorar la eficiencia económica y el crecimiento, así como mejorar la confianza de los inversores. El gobierno corporativo implica una serie de relaciones entre la dirección de la compañía, el consejo de administración, accionistas y el resto stakeholders" (OCDE, 2004).

En este sentido, dicho gobierno debe fortalecer los sistemas de gestión, control y administración de las empresas, constituyéndose como un instrumento de gestión entre accionistas y directivos. La base debe fundamentarse en los siguientes criterios: 1) otorgar un papel relevante y operativo a los consejeros independientes; 2) crear y potenciar el funcionamiento de los comités de auditoría, de nombramientos y retribuciones; 3 ) mejorar la transparencia en las sociedades y en las remuneraciones de los administradores, 4) favorecer los sistemas de control y otorgarles independencia.

Para las EES, la relación con sus distintos stakeholders cobra un especial interés, ya que la toma de decisiones en este tipo de entidades no depende tanto del capital aportado, sino de los intereses de los distintos agentes participantes (Puentes et al, 2009). Por ello, las EES llevan a cabo políticas socioeconómicas dirigidas a satisfacer y mejorar las condiciones del las partes implicadas y del entorno social y medioambiental donde se asientan (Bel y Ausin, 2007; Marcuello y Saz, 2008; Mozas y Bernal, 2006).

Con sus prácticas de buen gobierno contribuyen a la creación de empleo estable y de calidad (Jordan, 2002; Bel et al., 2005; Gomez et al., 2010 Servér y Capó, 2009; Puentes y Velasco, 2009; García, 20 I0) y al crecimiento de productividad empresarial (Benardi, 20l I). En este sentido, ayudan a atenuar los desequilibrios del mercado; además, estas organizaciones empresariales demuestran cierta cohesión social (Bel y Ausin, 2007), contribuyendo a integrar social o laboralmente a los distintos colectivos y al desarrollo del territorio en el que se asientan (Gallego, 2007; Mozas y Bernal, 2006).

Es posible afirmar que un gobierno corporativo en ES debe llevar a cabo políticas de buen gobierno encaminadas a, en primer lugar, contribuir con el desarrollo local del entorno social y medioambiental de la zona donde se establece la empresa, apostando por la mejora de la gestión y el conocimiento medioambiental $y$, en segundo lugar, favorecer la integración social e inserción laboral de colectivos en dificultades o con posibilidades de exclusión social, la creación de empleo estable y de calidad, generando riqueza para el entorno y aumentando la productividad de las EES del conocido como Tercer Sector.

\section{La importancia de la ES en España}

El número de EES en España ha ido incrementándose de forma muy notoria a lo largo de los últimos 
años. Según datos estadísticos del Ministerio de Trabajo y Asuntos Sociales, en 1999 el número total de sociedades cooperativas y sociedades laborales que era de 31 965 ha aumentado hasta alcanzar un total de 54788 en el 2007 (ver cuadro I).

Por sectores de actividad (ver gráfico I), el sector servicios es el que presenta más tasa de crecimiento, con un 88.33\%, pasando de 16623 empresas en 1999 a 31307 firmas en el 2007; el sector industrial, con un 35.86\%, pasando de 7048 empresas en 1999 a 9576 en el 2007, y el sector agrario, con un 5,9\% de aumento, pasando de 4046 empresas en 1999 a 4286 en el 2007.

\section{Impacto socioeconómico de las políticas de buen gobierno}

La creación de empleo estable y de calidad

Los distintos estudios analizados, tanto de CIRIECEspaña como de CEPES, coinciden que las EES son un motor importante para la creación de empleo empresarial de forma estable y de calidad, lo cual ha sido decisivo para el mantenimiento de más de dos millones de empleos en España, algo más del I0\% de la población laboral española (ver cuadro $n^{\circ} 2$ ). En este caso, los agricultores y ganaderos son los más numerosos, representados, en su mayoría, en organizaciones empresariales cooperativas, representando el $48.80 \%$ del total, seguido por los trabajadores autónomos, con un 17.80\%.

En cuanto a la calidad del empleo, los estudios empíricos demuestran que el empleo en las EES tiene una mayor calidad y estabilidad en comparación con el resto de entidades. Según datos de CEPES el $56.9 \%$ de los trabajadores en la ES ha tenido una o más experiencias de empleo continuadas, es decir, no ha tenido ningún episodio de desempleo; mientras que en las empresas capitalistas este porcentaje se reduce al $21.3 \%$, por lo que se puede reconocer que la estabilidad laboral es mayor en las EES.

\section{La integración social o laboral}

Una de las máximas de las EES, dirigida la implantación de sus políticas de buen gobierno, es la integración social o laboral de distintos colectivos, algunos de ellos con riesgo de exclusión social. Al comparar los datos de las EES con las sociedades anónimas (S.A.) y las sociedades limitadas (S.L.), vemos como, la integración colectivos muy afectados por el desempleo de larga duración (trabajadores mayores de 45 años) o colectivos de con riesgo de exclusión (personas con discapacidad) es mayor en las EES (ver cuadro 3) que en la S.A. y S.L.

En las EES, el porcentaje de las mujeres en plantilla es ligeramente menor que en las empresas ordinarias (35.9\% de mujeres en las plantillas frente a 39.2\%). Sin embargo, la participación de mujeres mayores de 45 años, en el empleo total, es mayor en las empresas de ES (un 15.39\% frente al 11.50).

En las EES también tiene una mayor presencia el colectivo de trabajadores mayores de 55 años, muy afectado por el desempleo de larga duración en comparación con el resto de entidades (13.8\% frente a 9.2\%).

Es interesante destacar los datos sobre la aportación de la ES a la hora de integrar a personas con discapacidad. El porcentaje medio de personas con discapacidad (grado superior al 33\%) en las EES es del $31.77 \%$ mientras que en las empresas capitalistas alcanza solo el $0.16 \%$.

Otro colectivo afectado por unas altas tasas de desempleo son los trabajadores con baja cualificación.

Cuadro I. Evolución del número de EES en España

\begin{tabular}{|l|c|c|c|c|c|c|c|c|c|c|}
\hline & 1999 & 2000 & 2001 & 2002 & 2003 & 2004 & 2005 & 2006 & 2007 \\
\hline Cooperativas & 22049 & 22930 & 23637 & 25041 & 25693 & 26877 & 27338 & 27435 & 27465 \\
\hline Total laborales & 9916 & 12787 & 15822 & 18954 & 20787 & 23198 & 25667 & 26913 & 27323 \\
\hline S.A.L. & 3872 & 3793 & 3751 & 3704 & 3629 & 3601 & 3568 & 3524 & 3473 \\
\hline S.L.L. & 6044 & 8994 & 12071 & 15250 & 17158 & 19597 & 22099 & 23389 & 23850 \\
\hline Total ES & 31965 & 35717 & 39459 & 43995 & 46480 & 50075 & 53005 & 54348 & 54788 \\
\hline
\end{tabular}


Gráfico I: Evolución del número de EES en España por sector de actividad

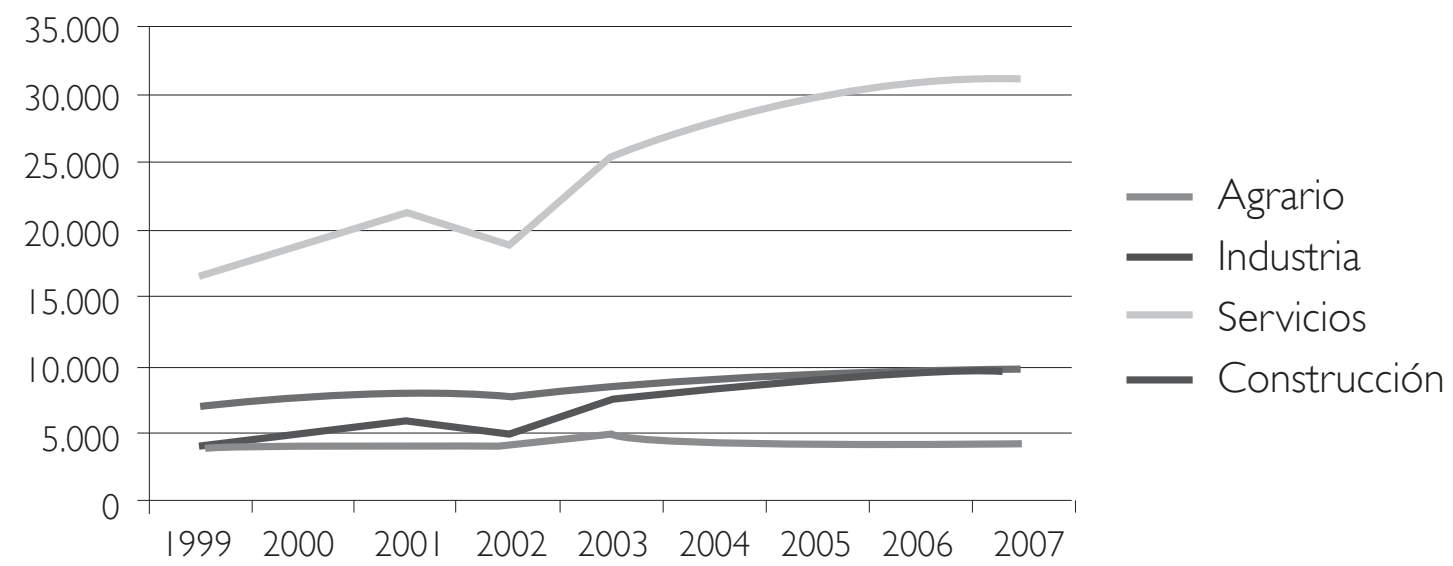

Fuente: Datos estadísticos del Ministerio de Trabajo e Inmigración.

Cuadro 2. Total de personas relacionadas con la ES en el 2010

\begin{tabular}{|l|r|r|}
\hline \multicolumn{1}{|c|}{ Personas relacionadas con las EES } & Número & \multicolumn{1}{c|}{$\%$} \\
\hline Agricultores y ganaderos asociados a cooperativas & I 160337 & $48.80 \%$ \\
\hline Autónomos & 42336 I & $17.80 \%$ \\
\hline Trabajadores en cofradías de pescadores & 442 & $0.02 \%$ \\
\hline Trabajadores en mutualidades & 1380 & $0.06 \%$ \\
\hline Trabajadores con discapacidad & 125157 & $5.26 \%$ \\
\hline Trabajadores en inserción & 1589 & $0.07 \%$ \\
\hline Asalariados en otras figuras jurídicas & 285360 & $12.00 \%$ \\
\hline Socios trabajadores y asalariados en sociedades laborales & 81772 & $3.44 \%$ \\
\hline Socios trabajadores/de trabajo y asalariados en cooperativas & 298514 & $12.55 \%$ \\
\hline
\end{tabular}

Fuente: Elaboración propia a partir de datos estadísticos de la Confederación Empresarial Española de la Economía Social.

Cuadro 3. Integración de colectivos con dificultades al empleo en el 2010

\begin{tabular}{|l|c|c|}
\hline & S.A.-S.L. & ES \\
\hline Mujeres & $39,20 \%$ & $35,90 \%$ \\
\hline Mujeres > 45 años & $11,50 \%$ & $15,30 \%$ \\
\hline Personas con 55 años y más & $9,20 \%$ & $13,80 \%$ \\
\hline Personas con discapacidad (<33\%) & $0,16 \%$ & $31,77 \%$ \\
\hline Entre el 33\% y el 65\% & $88,10 \%$ & $78,10 \%$ \\
\hline ggual o superior al 65\% & $11,90 \%$ & $21,90 \%$ \\
\hline
\end{tabular}

Fuente: Elaboración propia a partir de datos estadísticos de la Confederación Empresarial Española de la Economía Social (201 I). 
El grupo de peones y asimilados (en principio, los trabajadores con menor nivel de cualificación) representa casi un tercio, es decir el 32.3\%, de las plantillas en las empresas de ES, mientras que en las S.A y S.L. este porcentaje es del $12.5 \%$.

El desarrollo local

Una de las contribuciones de las EES al bienestar social y al desarrollo económico es su preocupación por el interés local de las zonas donde se asientan y el desarrollo económico de las zonas rurales contribuyendo al mantenimiento y desarrollo de estas zonas invirtiendo en ellas y generando empleo estable y de calidad apostando por políticas de desarroIlo local y generando riqueza (Gualati et al., 2000; Bueno et al., 2003: Briones, 2009; Gallego, 2007; Bel et al., 2005).

Los estudios analizados demuestran que la presencia de las EES en el ámbito rural es mayor que en las entidades capitalistas. Según CEPES, las EES se ubican mayoritariamente en municipios de ámbito rural, con población menor a 40000 habitantes: el $54.9 \%$ de sus empresas tiene su domicilio social en el ámbito rural y el $57.3 \%$ de sus trabajadores reside en uno de ellos.

En las empresas no pertenecientes a la ES, estos porcentajes se invierten y tanto, el domicilio de la sociedad como el de sus trabajadores se ubican mayoritariamente en el ámbito urbano (ver cuadro 4).

En relación con el domicilio de las empresas, es posible notar cómo las EES tienen una mayor presencia en las zonas rurales (57.30\%) que en las zonas urbanas (42.70\%). Dicha presencia es mayor que la de las empresas capitalistas en estas mismas zonas. La alta presencia de EES en tales lugares provoca un mayor aprovechamiento de las potencialidades económicas del ámbito rural siendo. Así, uno el aumento del empleo y de la actividad económica de las zonas rurales es uno de los principales efectos de las EES.

El establecimiento de empresas conduce a un aumento de la oferta de bienes y servicios en los espacios rurales necesario para abastecer el aumento de la población, la cual goza de estabilidad laboral y por tanto una mejor y más estable situación económica. La ES adquiere un mayor protagonismo en el desarrollo del tejido empresarial y productivo de la zona, donde tienen una importante presencia determinadas actividades sobre todo del ámbito agroalimentario.

\section{Conclusiones}

Desde el punto de vista de las implicaciones prácticas del buen gobierno corporativo, el desarrollo, la adopción y el cumplimiento de los códigos de buen gobierno está directamente relacionado con las estrategias de gobierno adoptadas por la empresa y en particular con las relaciones que el director tienen fuera y dentro de la empresa con los distintos stakeholders.

La evolución del concepto de gobierno ha ido muy ligada a la evolución de la sociedad y a los cambios en los mercados. Precisamente, la desconfianza en los mercados financieros acaecida en la última década ha provocado la aparición de una serie de códigos de buen gobierno corporativo necesarios

Cuadro 4. Domicilio social de las empresas y lugar de residencia de sus trabajadores por ámbito geográfico (20l0)

\begin{tabular}{|c|c|c|c|}
\hline \multicolumn{4}{|c|}{ Residencia de los trabajadores } \\
\hline & Ámbito urbano & Ámbito rural & \\
\hline Economía social & $45.10 \%$ & $54.90 \%$ & $100 \%$ \\
\hline Capitalistas & $56.20 \%$ & $43.80 \%$ & $100 \%$ \\
\hline \multicolumn{4}{|c|}{ Domicilio de la empresa } \\
\hline & Ámbito urbano & Ámbito rural & \\
\hline Economía social & $42.70 \%$ & $57.30 \%$ & $100 \%$ \\
\hline Capitalistas & $55.80 \%$ & $44.20 \%$ & $100 \%$ \\
\hline
\end{tabular}

Fuente: Elaboración propia a partir de datos estadísticos de la Confederación Empresarial Española de la Economía Social $(2011)$. 
para sentar unas bases éticas y marcar pautas de conducta en el gobierno de la empresa, códigos que aunque se dirigen a las empresas capitalistas pueden ser adaptados a las EES.

Es tanta su importancia que los gobiernos están redactando códigos de buenas prácticas y trasparencia para las administraciones púbicas, como en el caso de España en donde se ha aprobado el Anteproyecto de Ley de Transparencia, Acceso a la Información Pública y Buen Gobiernol destinado a todas las administraciones. Este código tendrá rango de ley, por primera vez en la historia.

Se puede afirmar que la aplicación de políticas de buen gobierno enmarcadas en los postulados de la ES lleva consigo un mayor desarrollo social y económico del entorno en donde las entidades actúan. Esto favorece la integración social y laboral de colectivos con dificultades al crear empleo estable y de calidad, genera riqueza y crecimiento económico y aumenta la productividad de las EES del conocido Tercer Sector y de la sociedad en general.

\section{Bibliografía}

Bel, P. \& Ausin, J.M. (2007): Contribución de las sociedades cooperativas al desarrollo territorial. En REVESCO. N 92, pp. $4|-7|$.

Bel. P., Fernández J. \& Miranda, M. (2005). La creación de puestos de trabajo en el ámbito rural para su desarrollo: las organizaciones de participación agrarias. En CIRIEC-España, Revista de Economía Publica, Social y Cooperativa. No 52, pp. 335-360.

Benardi, A. (20 I I). El mercado de trabajo y el sector cooperativo en Italia. En CIRIEC-España, Revista de Economía Publica, Social y Cooperativa. N 70, pp. I49- 178.

Briones A. J. (2009). Crecimiento en cooperación en las Entidades de economía Social: creatividad, innovación y responsabilidad. En: XII Jornadas de investigadores en economía social y cooperativa, CIRIEC-España y UCOMUR, Murcia.

Bueno Campos, E. Coordinador (2004). El gobierno de la empresa. Madrid: Ediciones pirámide.

Bueno Campos, E; Rodríguez, O. \& Salmador, M.P. (Septiembre, 2003). El capital social en el capital intelectual de las organizaciones: propuesta y contraste empírico de un modelo de medición y gestión. En XIII Congreso Nacional ACEDE, Salamanca.Chaves, R. (2004). Gobierno y democracia en las Entidades de Economía Social. Mediterráneo económico. $N^{\circ}$ 6, p. 35-52.
Gallego, L. (2007). Función de las cooperativas en la normativa de fomento del desarrollo rural. REVESCO. No 92, Segundo Cuatrimestre, pp. 153-177.

Garcia, J. (2010). La necesidad de armonización internacional del derecho cooperativo: el caso Español. REVESCO. NoI02, Segundo Cuatrimestre, pp. 79- 108.

Gomez, J.M., Roman Ma A. \& Rojo, C. (20 I0). Las dimensiones de la calidad y el empleo en la economía social un análisis de las Sociedades Laborales y de los Centros Especiales de Empleo en Castilla y León. En CIRIEC-España, Revista de Economía Publica, Social y Cooperativa. No67, pp. 45-74.

Gualati, R., Noria, N. \& Zaheer, A. (2000): Strategic networks, Strategic Management Journal, $n^{\circ} 21$, pp. 203-2I 5.

Jordan, R. (2002). Particularidades de las condiciones Laborales de en las empresas de trabajo asociado. CIRIEC-España, Revista de Economía Pública, Social y Cooperativa. N 42, pp. 33-47.

Juliá, J.F., (2004). La economía social y el cooperativismo, la democracia en la economía. A modo de introducción. En Mediterráneo económico. № 6, p.10-14.

Marcuello, C. \& Saz, I., (2008). Los principios cooperativos facilitadores de la innovación: un modelo teórico. En REVESCO. No 94, Primer Cuatrimestre, pp. 59-79.

Mozas, A. \& Bernal, E. (2006). Desarrollo territorial y economía social. En CIRIEC-España, Revista de Economía Publica, Social y Cooperativa. No55, pp. 125-140.

Organización para la Cooperación y el Desarrollo Económico (OCDE). Guidelines of multinational enterprice. París: 2004.

Puentes, R., Velasco, Ma.M., Villar, J. (2009). El buen gobierno corporativo en las sociedades cooperativas. REVESCO. № 98, Segundo Cuatrimestre, pp. I |8-140

Puentes R., \& Velasco, M M. (2009). Importancia de las sociedades cooperativas como medio para contribuir al desarrollo económico, social y medioambiental, de forma sostenible y responsable. En REVESCO, No 99, Tercer Cuatrimestre, pp. 104-129.

Saz, Ma I. \& Carús, L. (2008). Los procesos participativos en la sostenibilidad medioambiental. El caso del segmento turístico recreativo de Alta Montaña. En CIRIEC-España, Revista de Economía Pública, Social y Cooperativa. Nº 6I, pp. 127-I56.

Seguí, E., García, G., Romero, A. \& Villalonga I. (2008). La Innovación en el gobierno de las cajas rurales españolas: evaluación de su e-gobierno corporativo. En CIRIEC-España, revista de Economía Publica, Social y Cooperativa. No 60, abril 2008, pp. I55- 178.

Servér, R.J. \& Capó,J. (2009). La Responsabilidad Social Empresarial en un contexto de crisis. Repercusión en las Sociedades Cooperativas. En CIRIEC-España, Revista de Economía Publica, Social y Cooperativa. $n^{\circ}$ 65, agosto, pp. 7-31.

I Fue aprobado por el Consejo de ministros en marzo del 2012. Puede ser consultado en la página www.leydetransparencia.gob.es 